and Riddle and Roth (1970) pursued this idea and the second group isolated several strains of Salmonella typhimurium carrying such external suppressors. And in the current issue of the Journal of Molecular Biology (66, 483 and 494 ; 1972) Riddle and Roth report, first, the genetic mapping of these frameshift suppressor mutations and, second, their association with altered tRNA species.

Riddle and Roth's collection of suppressors map at six distinct and widely separated loci defining six suppressor genes (sufA-F) and the location of the sufD gene on the $S$. typhimurium chromosome corresponds to the location of a glycine tRNA gene on the Escherichia coli chromosome. The genetic maps of these two species are very similar and so from the genetic data alone it would not be surprising if the sufD gene proved somehow to be related to a glycine tRNA gene. The fact that four of the suppressor mutations, suf A, B, D and E, are dominant to the corresponding wild type genes when these are introduced on an $\mathrm{F}^{\prime}$ cpisome is also, of course, consonant with the idea that these genes may specify altered tRNAs.

The crucial test of such an idea is, however, the isolation from the mutant strains of tRNAs capable of frameshift suppression in vitro. Riddle and Roth have gone some way towards this goal by showing that in strains carrying either the sufA or sufB genes two proline tRNAs have changed mobilities on benzoylated DEAEcellulose columns, while the suf $\mathrm{D}$ gene is associated with an altered glycine tRNA and the sufF gene is associated with an increased heterogeneity of glycine tRNAs.

Riddle and Roth have not managed so far to associate the sufC and sufE genes with altered tRNAs, but that does not, of course, rule out the idea that these suppressors act by way of tRNA. In short, there seems little doubt that at least some external frameshift suppressor mutations cause changes in tRNAs and they may well be located in the structural genes of those molecules. It will, of course, be most interesting to learn the base sequences of the altered $\operatorname{tRNA}$, for that information may give clues to the mechanism of suppression, which, it is widely supposed, involves tRNAs reading quadruplets instead of triplets of bases in regions of an RNA where one base is repeated several times and thereby restoring the reading frame.

Everybody working with frameshift suppressors has envisaged suppression by tRNAs because of the amber and ochre suppressor precedents. It is not surprising therefore that Gorini and his colleagues should have searched, with success, for mutations of ribo-

somal proteins which also lead to the suppression of frameshift mutations. Atkins, Elseviers and Gorini, for example, have just reported (Proc. US Nat. Acad. Sci., 69, 1192; 1972) that the leakiness of sixteen different frameshift mutations in the lac operon of $E$. coli is controlled by the ribosome. In cells with wild type ribosomes some $\beta$-galactosidase is made in spite of the frameshift mutations. But when the strA mutation, which alters the P10 protein of the $30 \mathrm{~S}$ ribosomal subunit and also restricts errors and ambiguity of translation, is introduced the amount of $\beta$-galactosidase made, the leakiness in other words, is cut to barely a fifth of its previous level. Moreover, when the ram mutation is also introduced (the ram mutation alters the $\mathrm{P} 4 \mathrm{a}$ ribosomal protein and antagonizes the restriction of the strA mutation) the amount of $\beta$-galactosidase made, at lcast in some cases, is restored to the levels detectable in cells with wild type ribosomes.

These data indicate that the structure of the ribosome has a role in the suppression of frameshift mutations presumably by promoting or restricting the frequency of translation of quadruplets of bases by wild type tRNAs. If this idea is correct one might predict that cells carrying frameshift suppressor mutations affecting tRNAs as well as the ram mutation affecting the ribosomes would suppress frameshift mutations with very high efficiencies.

\title{
Different Scrapie Agents Compete
}

IN next Wednesday's Nature New Biology (June 21) Dickinson, Fraser, Meikle and Outram report a series of experiments which leave no room to doubt that different strains of scrapie agent simultaneously infecting the same mouse interfere competitively with each other's replication. Dickinson and his colleagues have used for their experiments VM mice as hosts and the 22A and $22 \mathrm{C}$ strains of scrapie agent. In VM mice $22 \mathrm{C}$ strain has a much longer incubation time than has $22 \mathrm{~A}$ and the pattern of brain lesions caused by these two strains differs such that in doubly infected VM mice $22 \mathrm{~A}$ and $22 \mathrm{C}$ lesions can be distinguished.

Dickinson et al. set out to discover whether these two strains of scrapie agent act synergistically or competitively when both are inoculated in the brain or peritoneum of a host. Because the $22 \mathrm{C}$ strain from C57Bl mice replicates so much more slowly than does the $22 \mathrm{~A}$ strain from VM mice, it was necessary to inoculate the VM host with the $22 \mathrm{C}$ strain first and then challenge afterwards with the $22 \mathrm{~A}$ strain.

From such experiments Dickinson et
APPLIED ECOLOGY

Computerized Ecosystem

from a Correspondent

RESEARCH into the complex interrelationships within ecological systems has received considerable impetus from the development of analogue computer techniques. The cycling of minerals within ecosystems has proved a particularly suitable field for the application of systems analysis and the consequent generation of predictive models. In the current issue of the Journal of Applied Ecology (9, 25; 1972) P. F. May, A. R. Till and M. J. Cumming (CSIRO, Australia) describe the application of such techniques to the study of the movement of sulphur in sheep-grazed pastures and its final accumulation in wool.

Data for the model have been col. lected during the past eight years and chiefly involve the use of sulphur-35 as a radioactive tracer. Sulphur was initially applied to an experimental grassland in New South Wales in 1964 as ${ }^{35}$ S-gypsum and its movement was followed during the course of 760 days. The rates of incorporation of the tracer were measured in the soil, in plant tissue, in the ingested herbage (measured by fistulation), in the wool and in the excreta of the sheep. These rates, in May et al.'s opinion, represent the major pools within the system and are easily measurable. Other subsidiary pools, such as the soil organic matter and the

al. find that, if the $22 \mathrm{C}$ intracerebral injection precedes by some 5-9 weeks the $22 \mathrm{~A}$ injection, the incubation period of the 22A-induced brain lesions is increased by at least 30 days. An injection of $22 \mathrm{C}$ agent one week before an injection of $22 \mathrm{~A}$ agent does not, by contrast, prolong the latent period.

From these and other observations Dickinson et al. reach several tentative conclusions. First, it seems likely that the slowly replicating $22 \mathrm{C}$ agent may compete with the $22 \mathrm{~A}$ agent and slow its replication by blocking a significant proportion of putative sites at which the agents can replicate. Second, the slow replication of the $22 \mathrm{C}$ strain and the failure to observe competition against the $22 \mathrm{~A}$ strain when the interval between the two injections is short suggests that at least in VM mice the 22C strain may have to be somehow modified or processed before it can bind to a significant number of the putative replication sites. Third, there is no evidence of any synergism between these two strains and the competition that is observed is unlikely to involve an orthodox immune response. 